\section{CXCR4 as a prognostic marker in Egyptian chronic lymphocytic leukemia patients}

\author{
Wafaa T El-sherif ${ }^{1}$, Eman M Salah El-din ${ }^{1}$, Mai M. \\ Aly $^{2}$, Mohamed G Said ${ }^{1}$, Dalia A Nigm ${ }^{1}$
}

${ }^{1}$ Clinical Pathology Department, Faculty of Medicine, Assiut University, Assiut, Egypt.

${ }^{2}$ Clinical Hematology Unit, Faculty of Medicine, Assiut University, Assiut, Egypt.
The Egyptian Journal of Immunology Volume 28 (3), 2021: 114-126. www.Ejimmunology.org

\begin{abstract}
Chronic lymphocytic leukemia (CLL) has variable clinical presentations, and molecular and biological prognostic markers. The C-X-C chemokine receptor 4 (CXCR4) and its ligand stromal cell-derived factor-1 (SDF-1) play an important role in trafficking of lymphocytes and monocytes. The aim was to study lymphocyte expression of CXCR4 and its prognostic value in CLL. A case control study was carried out on 30 newly diagnosed CLL cases and 30 healthy controls. Fludarabine, cyclophosphamide, and rituximab (FCR) was the standard treatment. Flowcytometric measurement of CXCR4 expression on lymphocytes was done. CXCR4 was significantly higher in patients than controls $(81.67 \pm 17.95$ vs. $11.78 \pm 2.78 ; P<0.001)$. CXCR4 was significantly higher $(P<0.001)$ in high risk CLL $(93.63 \pm 6.78) v s$. intermediate risk $(82.50 \pm 7.13)$ and low risk $(75.84 \pm 12.23)$. CXCR4 was significantly higher $(P<0.001)$ in non-responders $(91.63 \pm 6.98)$ vs. partial responders $(83.11 \pm 5.55)$ and complete responders (70.11 \pm 4.44$)$. CXCR4 was significantly lower in survivors vs. non-survivors (80.89 \pm 5.09 vs. $85.43 \pm 5.51 ; P<0.001$. CXCR4 had significant positive correlation with WBCs $(r=0.45$, $P=0.01)$ and lymphocytes $(r=0.40, P=0.01)$ measured at diagnosis. In conclusions, expression of CXCR4 in newly diagnosed CLL is significantly high. CXCR4 increased expression is associated with poor prognosis and resistance to the therapy.so it can be used as prognostic tool.
\end{abstract}

Keywords: CLL, CXCR4 expression, prognosis.

Date received: 19 February 2021; accepted: 22 May 2021

\section{Introduction}

Chronic Lymphocytic Leukemia (CLL) is a disease of accumulation of numerous number of clonal $\mathrm{CD}^{+} \mathrm{B}$ lymphocytes in peripheral blood, lymph nodes, bone marrow (B.M) and spleen. ${ }^{1}$

The exact pathogenesis of CLL is promoted. CLL is an acquired disorder, and reports of truly familial cases are very rare. ${ }^{2}$ The most frequent chromosomal abnormality in CLL is deletion of
$13 q 14$ which occurs in more than $50 \%$ of patients, being monoallelic in $76 \%$, and biallelic in $24 \%$ of cases. This deletion, also detected in Monoclonal B cell Lymphocytosis (MBL), found at a much lower frequency in multiple myeloma (MM), diffuse large B-cell lymphoma (DLBCL), mature T-cell lymphomas, and in various solid tumors. $^{3}$

CXCR4 (chemokine C-X-C motif receptor 4), also known as CD184, is a chemokine $G$ protein 
coupled receptor, expressed in various cell species, including normal B lymphocytes. CXCR4 is overexpressed in a multiple number of malignancies including CLL, Acute Myeloid Leukemia ( $A M L), M M$, lymphomas, and solid tumors. CXCL12 (chemokine C-X-C motif ligand 12), also known as stromal cell-derived factor 1 (SDF-1), is CXCR4 sole ligand. It is a homeostatic chemokine, expressed in high rate in lymph nodes, B.M, liver, and lung. CXCL12 regulates hematopoietic cell trafficking and their homing to the BM. Chemotaxis driven by CXCR4 and CXCL12 interactions has been proved to control various biological functions including cell adhesion, migration, and invasion. ${ }^{4}$ These observations have led to the suggestion that CXCR4 expressed on B-CLL cells plays a major role for heterotypic adherence to marrow stromal cells and contributes to the tropism and infiltration of B-CLL cells to the B.M. In addition, in other types of cancer, the CXCR4/SDF-1 ligand has been postulated to play a role in tumor cell migration and metastatic destination, including the B.M. ${ }^{5}$

The CXCR4-CXCL12 axis is an important therapeutic target in CLL, Burger M, et al 2005 demonstrated that small peptide CXCR4 antagonists effectively block CXCL12-induced activation, migration and signaling of CLL cells. ${ }^{6}$

Stromal cell-mediated protection from spontaneous or fludarabine based regimens induced apoptosis of CLL cells was partially blocked, suggesting a potential role of CXCR4 antagonists in combination with a B-cell targeted therapy in the treatment of CLL. In CLL, CXCR4-expressing cancerous B-cells are attracted toward bone marrow stromal cells, which secrete high levels of CXCL12, resembling the homing of normal hematopoietic stem cells to bone marrow. Inhibition of CXCR4 was able to decrease cancer cells growth and metastasis, and studies on its mechanism mainly focused on protein kinase $B$ (AKT) and mitogen-activated protein kinases (MAPK) signaling pathway. ${ }^{7}$

The main aim of this study is to evaluate the percentage of CXCR4 in the CLL leukemic cells by flow-cytometer, to describe the presence of CXCR4 in both low and high risk CLL as well as in responders and non-responders to fludarabine, cyclophosphamide, and rituximab (FCR) therapy in CLL patients.

\section{Patients and Methods}

This study included $30 \mathrm{CLL}$ patients and 30 age and sex- matched healthy controls. The patients were selected from Clinical Hematology Unit, Internal Medicine department and outpatient clinic over a period 2 years from April 2017 to May 2019. All patients provided approved informed consent. Patients were newly diagnosed as CLL after Bone marrow aspirate (BMA), and Immunophenotyping. Patients were enrolled before treatment, and followed up for 6 month by complete blood count to assess their response to FCR regimen. ${ }^{8,9}$ Formal consent was obtained from patients and controls. The study was approved by Ethical Committee of Faculty of Medicine Assiut University.

All patients were subjected to history taking and clinical examination stressing on the presence of lymphadenopathy, splenomegaly, hepatomegaly, and symptoms and signs of anemia and/or thrombocytopenia. Imaging studies were performed for assessing organomegaly. Patient were classified according to modified Rai Classification which defines lowrisk disease as patients who have lymphocytosis with leukemia cells in the blood and/or marrow (lymphoid cells > 30\%; formerly considered Rai stage 0). Patients with lymphocytosis, enlarged nodes in any site, and splenomegaly and/or hepatomegaly (lymph nodes being palpable or not) are defined as having intermediate risk disease (formerly considered Rai stage I or stage II). High-risk disease includes patients with disease-related anemia (as defined by a hemoglobin [Hb] level <110 g/L $[11 \mathrm{~g} / \mathrm{dL}]$; formerly stage III) or thrombocytopenia (as defined by a platelet count $<100 \times 10^{9} / \mathrm{L}$; formerly stage IV). ${ }^{10}$ Patients' response classified to complete response, partial response and progressive disease according to iwCLL guidelines. ${ }^{11}$

Complete response criteria include: peripheral blood lymphocytes (evaluated by blood and differential count) $<4 \times 10^{9} / \mathrm{L}$. Absence of significant lymphadenopathy by 
physical examination. In clinical trials, a CT scan of the neck, abdomen, pelvis, and thorax is desirable if previously abnormal. Lymph nodes should be, $1.5 \mathrm{~cm}$ in longest diameter. No splenomegaly or hepatomegaly by physical examination. Absence of disease-related constitutional symptoms. Blood counts need to show the following values: Neutrophils $>1.5 \times$ $10^{9} /$ L. Platelets $>100 \times 10^{9} /$ L. Hemoglobin $>11.0$ $\mathrm{g} / \mathrm{dL}$ (without red blood cell transfusions).

Partial response criteria: A decrease in the number of blood lymphocytes to $50 \%$ or less from the value before therapy. Reduction in lymphadenopathy compared with baseline (by cross-sectional imaging scans in clinical trials or by palpation in general practice) as defined by: A decrease in lymph node size by $50 \%$ or more in the sum of the products of the same enlarged lymph nodes selected at baseline as assessed by imaging (an established number in clinical trials of lymph nodes has been up to 6), and the sum of longest diameters of the same enlarged lymph nodes selected at baseline as assessed by physical examination (an established number in clinical trials of lymph nodes has been a maximum of 6). No increase in any lymph node and no new enlarged lymph node (diameter $>1.5 \mathrm{~cm}$ ). A regression $>50 \%$ of the extent of enlargement of the spleen below the costal margin defined by palpation, or normalization in size. When assessed by $\mathrm{CT}$, scan spleen size must have regressed by $>50 \%$ in length beyond normal. A persistence of splenomegaly post therapy may have limited influence on outcome in CLL. A regression of $>50 \%$ of the extent of enlargement of the liver below the costal margin defined by palpation, or normalization in size. The blood count should show 1 of the following results: Platelet counts $>100 \times 10^{9} / \mathrm{L}$ or $50 \%$ improvement over baseline. $\mathrm{Hb}>11.0$ $\mathrm{g} / \mathrm{dL}$ or $50 \%$ improvement over baseline without red blood cell transfusions or erythropoietin support.

Progressive disease characterized by one of the following criteria: Disease progression occurs if 1 of the following events is observed. Appearance of any new lesion such as enlarged lymph nodes $(1.5 \mathrm{~cm})$, splenomegaly, hepatomegaly, or other organ in- filtrates. An increase in the spleen size by $50 \%$ or the de novo appearance of splenomegaly. An increase in the liver size of $50 \%$ of the extent enlargement of the liver below the costal margin defined by palpation, or the de novo appearance of hepatomegaly. An increase in the number of blood lymphocytes by $50 \%$ or more with at least $5 \times 10^{9} / \mathrm{L}$ B lymphocytes. Transformation to a more aggressive histology (Richter syndrome or Richter transformation). The diagnosis of Richter transformation should be established by lymph node or other tissue biopsy. Occurrence of cytopenia (neutropenia, anemia, or thrombocytopenia) directly attributable to CLL and unrelated to autoimmune cytopenias.

\section{Routine laboratory tests}

We performed the routine lab workup for CLL patients as follow: six $\mathrm{ml}$ of venous blood were collected under complete aseptic conditions and divided into:

-Two $\mathrm{ml}$ of venous blood in sodium citrate containing tube for prothrombin time and concentration done on Sysmex CA- 1500 System (Siemens, Germany).

-Two $\mathrm{ml}$ were collected into plain tube without anti-coagulant for kidney function and liver function COBAS Integra 400 plus, Roche (Germany) and LDH on Dimention (Siemens, Germany).

-Two $\mathrm{ml}$ of venous blood were collected into EDTA containing tube and used immediately for CBC with spreading of PB smears for Leishman staining and differential leucocytic counting by ABBOTT Cell DYN 1700 Hematology Analyzer, USA and ADVIA (2120) laying stress on lymphocyte percentage, and absolute lymphocyte count. ${ }^{12}$ BMA was done for each patient from the anterior or posterior superior iliac spine. Small amount of BMA (less than 0.5 $\mathrm{ml}$ ) was mixed immediately on glass slides and smears were spread to be examined after staining by Leishman stain. Another one $\mathrm{ml}$ of BMA was dispensed gently into a tube containing K-EDTA solution to be used for Flowcytometric (FCM) immunophenotypic analysis and the lab routine and flowcytometry was done by clinical pathologist team in study. 
The sample used for FCM was evaluated immediately or stored at room temperature $\left(18-22^{\circ} \mathrm{C}\right)$ to be used within 24 hours.

BM aspiration was used to prepare Leishman-stained smears for determination of lymphocyte percentage and morphology. ${ }^{13}$

\section{Immunophenotyping}

Bone marrow samples were subjected to immunophenotyping (Chronic lymphoproliferative panel). It includes CD5, CD10, CD19, CD4, CD8, CD3, CD16, CD56 as the primary panel and $k / \lambda / C D 19 /, F M C 7 / C D 23 /$ CD19, CD38/CD79b, slgm/CD19, CD20/CD200 as the secondary panel ${ }^{14,15}$ using BD FACS Calibur Flow Cytometer from Becton Dickenson (Indianapolis, Indiana 46268, United States)and the analysis was done to confirm CLL diagnosis and fulfilling criteria of CD5 positive lymphoproliferative disorders

\section{Flowcytometric analysis for CXCR4 expression}

Monoclonal antibody ( $\mathrm{mAb}$ ) reacts with human CXCR4, also known as CD184. The PE-CD184 was obtained from (Beckman Coulter Company, France). First, $100 \mu \mathrm{L}$ of specimen was pipetted in tubes labeled with the patient's name and the relevant mAb. Next, $10 \mu \mathrm{L}$ of appropriate fluorochrome-conjugated $\mathrm{mAb}$ was added and mixed thoroughly. The tubes were incubated in the dark at room temperature for $15 \mathrm{~min}$. The contents were mixed well and $1 \mathrm{~mL}$ of lysing solution was added to lyse the RBCs. The tubes were once more incubated in the dark at room temperature for $15 \mathrm{~min}$. Centrifugation was performed for $5 \mathrm{~min}$ at $2000 \mathrm{rpm}$, and the supernatant was discarded. The cells were washed with PBS, and the cell pellet was resuspended in 200-500 $\mu \mathrm{L}$ of PBS. Data were acquired on the flowcytometry.

Two color flowcytometric analysis dot plots and quadrant statistics were generated. In the first dot plot a region around the lymphocyte population on a forward scatter versus side scatter dot plot was created manually. Lymphocytes were gated for further analysis of the studied mAbs, and the gated positive cells were $77.7 \%$ of the total bone marrow mononuclear cells Figure 1a, a marker was considered positive if the percentage of positive cells was more than $20 \%$ of the gated bone marrow mononuclear cells. From the gated lymphocytes, the percent of CD 19 positive lymphocytes was $92.6 \%$ and the percent of $C D$ 200 Was $95 \%$ of the gated cells Figure $1 \mathrm{~b}$. From the gated cells the percent of CD5, CD19 double positive cells were $94 \%$ Figure 1c., while, the percent of CD-184 positive cells was $97 \%$ of the gated B-lymphocyte Figure 1d.

\section{Statistical analysis}

Data was collected and analyzed those using SPSS (Statistical Package for the Social Science, version 20, IBM, and Armonk, New York). Continuous data was expressed in form of mean \pm SD or median (range) while nominal data was expressed in form of frequency (percentage). $\mathrm{Chi}^{2}$-test was used to compare the nominal data of different groups in the study while student $t$ test was used to compare mean of different two groups and ANOVA test for more than two groups. Person correlation was used to determine the correlation between level of CXCR4 and other continuous variables. Level of confidence was kept at $95 \%$ and hence, $P$ value was considered significant if $<0.05$. 
$a$

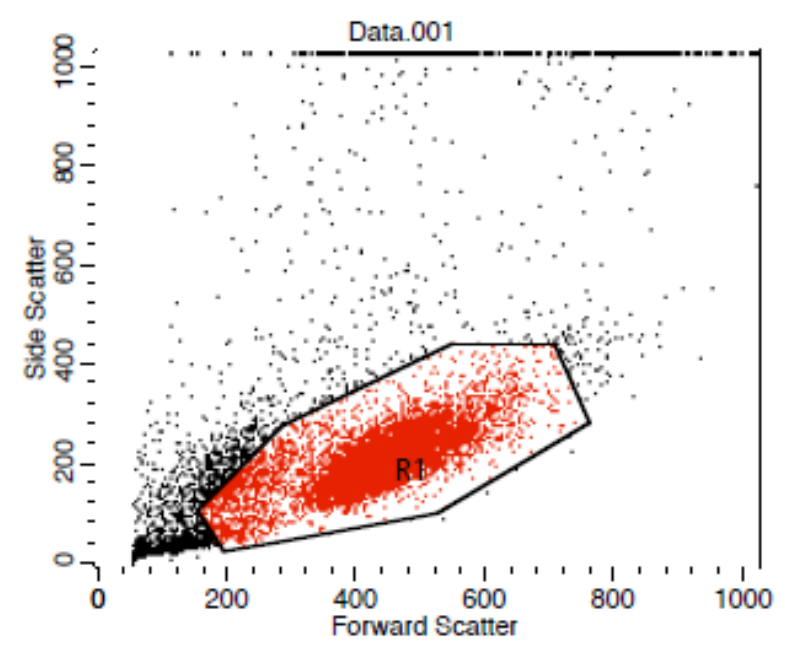

b

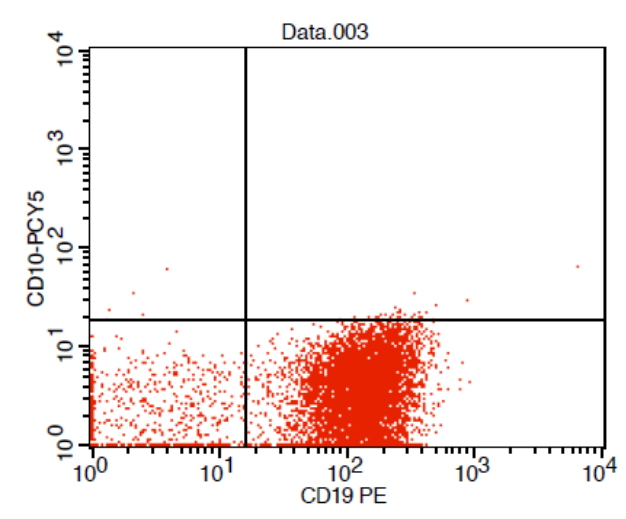

C

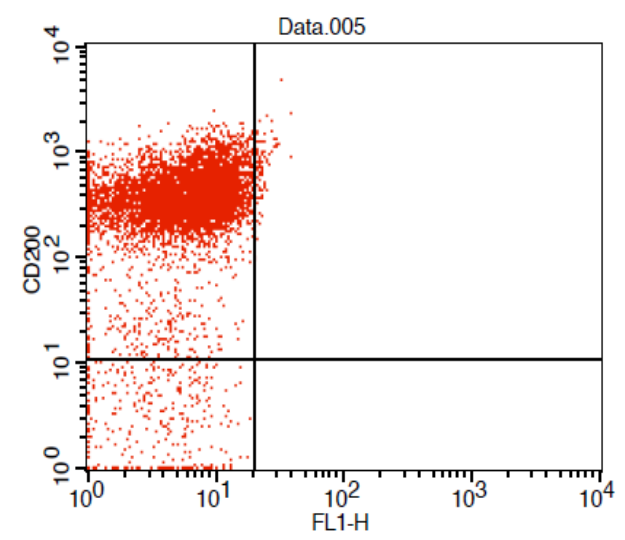

d
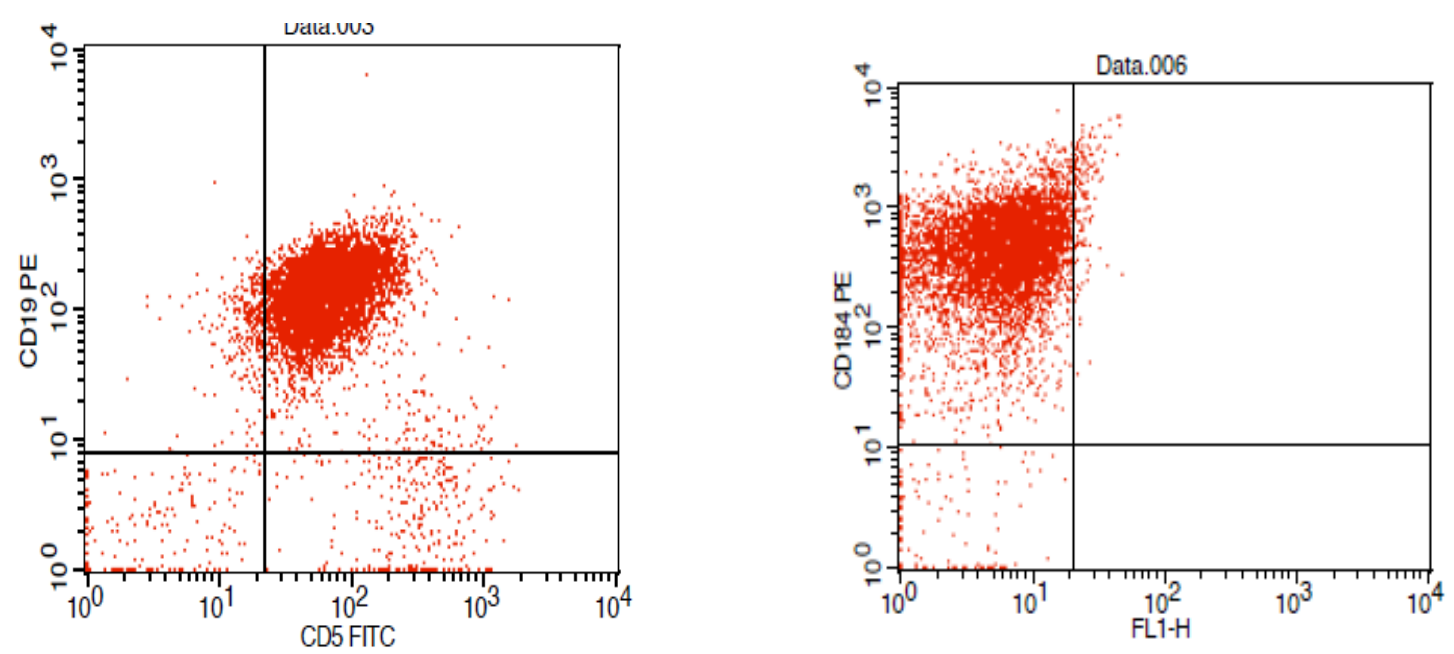

Figure 1. Flow cytometric analysis. (a) Using typical forward and side scatter characteristics, the gate was $77.7 \%$ from the total bone marrow mononuclear cells (b) From the lymphocyte gate, the percent of CD 19 is $92.6 \%$ from the gated cells and the percent of CD 200 is $95 \%$ from the gated cells. (c)From the gated cells the percent of CD5, CD19 double positive cells were 94\%. (d) From the lymphocyte gate, percent of CD 184 positive cells $97 \%$ from the gated B-lymphocyte. 


\section{Results}

\section{Patients' characteristics}

The age \pm (SD) of studied patients $(n=30)$ was $60.73 \pm(10.42)$ years with range between 33 and 80 years. Twenty-three $(76.7 \%)$ were males and $10(33.3 \%)$ were smokers Table 1 . Table 2 shows the clinical manifestations of studied patients. Constitutional symptoms were the frequent manifestation where 24 (80\%) patients suffered from fatigue followed by 14 patient fever (46.7\%), and lymphadenopathy in 13 patients $(63.3 \%)$, splenomegaly in 12 patients $(40 \%)$, bleeding tendency in 10 patients (33.3\%) and hepatomegaly in 9 patients (30\%).LN and splenomegaly and lymphadenopathy by examination. Table 3 shows the hematological laboratory data of enrolled patients.

In this study, modified Rai staging was distributed as follows: $9(30 \%)$ patients were classified as high risk CLL, 6 (20\%) patients were classified as intermediate risk CLL and 15 (50\%) patients were classified as low risk Figure 2a.

Table 1. Demographic data of patients with CLL.

\begin{tabular}{lc}
\hline Variables & $\mathrm{n}=30$ \\
\hline Age (years) Mean age \pm (SD) & $60.73 \pm 10.42$ \\
\hline Range & $33-80$ \\
\hline Sex & \\
$\quad$ Male \% & $23(76.7 \%)$ \\
$\quad$ Female \% & $7(23.3 \%)$ \\
\hline Smoker \% & $10(33.3 \%)$ \\
\hline Nonsmokers \% & $20(66.7 \%)$ \\
\hline $\begin{array}{l}\text { Data were expressed in the form of mean } \pm S D \text { and } \\
\text { frequency (\%). }\end{array}$
\end{tabular}

Table 2. Clinical data of enrolled patients with CLL.

\begin{tabular}{lc}
\hline Variables & $\mathrm{n}=30$ \\
\hline Constitutional symptoms & $24(80 \%)$ \\
Lymadenopathy & $13(43.3 \%)$ \\
Splenomegaly & $12(40 \%)$ \\
Bleeding tendency & $10(33.3 \%)$ \\
Hepatomegaly & $9(30 \%)$ \\
\hline Data was expressed in form of frequency (percentage).
\end{tabular}

Table 3. Hematological data of the enrolled patients with CLL.

\begin{tabular}{lc}
\hline Parameters & Mean \pm SD \\
\hline Complete blood picture & \\
\hline \multicolumn{1}{l}{ Hemoglobin $(\mathrm{g} / \mathrm{dl})$} & $10.64 \pm 1.57$ \\
\hline Platelets $\left(\times 10^{3} / \mu \mathrm{l}\right)$ & $171.98 \pm 62.36$ \\
\hline \begin{tabular}{l} 
White blood cells $\left(\times 10^{3} / \mu \mathrm{l}\right)$ \\
\hline $\begin{array}{l}\text { Relative count of Neutrophil } \\
\%\end{array}$
\end{tabular} & $14.58 \pm 8.16$ \\
\hline $\begin{array}{l}\text { Relative count of } \\
\text { Lymphocytes \% }\end{array}$ & $78.88 \pm 12.45$ \\
\hline $\begin{array}{l}\text { Relative count of Monocytes } \\
\%\end{array}$ & $3.22 \pm 1.11$ \\
\hline $\begin{array}{l}\text { Relative count of Eosinophil } \\
\%\end{array}$ & $2.01 \pm 0.11$ \\
$\quad$ Reticulocyte \% & $1.44 \pm 0.98$ \\
\hline Data was expressed in form of frequency (percentage).
\end{tabular}

\section{Bone Marrow Study for Enrolled Patients}

Table 4 shows bone marrow findings in enrolled patients. There were depressed myeloid precursors in 29 patients (96.7\%), erythroid precursors in 24 patient $(80 \%)$ and megakaryocytes in 15 patients (50\%). The most dominant cells in bone marrow were lymphocytes $(75.17 \pm 10.98 \%)$ followed by prolymphocyte $(5.09 \pm 0.99)$.

Table 4. Bone marrow study of enrolled patients with CLL.

\begin{tabular}{lc}
\hline Variables & $\mathrm{n}=30$ \\
\hline $\begin{array}{l}\text { Depressed precursors } \\
\text { Myeloid }\end{array}$ & $29(96.7 \%)$ \\
Erythroid & $24(80 \%)$ \\
Megakaryocytes & $15(50 \%)$ \\
\hline Differential cells & \\
Blast (\%) & $0.95 \pm 0.11$ \\
Promyelocyte (\%) & $0.91 \pm 0.32$ \\
Myelocyte (\%) & $1.33 \pm 0.33$ \\
Juvenile (\%) & $1.19 \pm 0.89$ \\
Band (\%) & $1.15 \pm 0.91$ \\
Neutrophils (\%) & $4.99 \pm 1.75$ \\
Lymphocyte (\%) & $75.17 \pm 10.98$ \\
Prolymphocyte (\%) & $5.09 \pm 0.99$ \\
Eosinophil (\%) & $2.01 \pm 0.75$ \\
\hline Data were expressed in the form of mean \pm SD and \\
frequency (\%).
\end{tabular}




\section{Effect of treatment on leucocytes}

After therapy, there was significant reduction in level of leukocyte count ( $90.25 \pm 14.78$ vs. 14.85 $\pm 8.16 ; P=0.03)$ and relative lymphocytic count (78.88 \pm 12.45 vs. $11.88 \pm 20.68 ; P=0.04$ ) while hemoglobin and platelets showed insignificant changes Figure $2 b$.

a

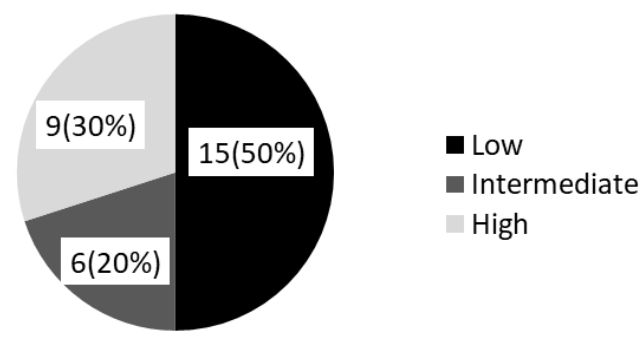

b

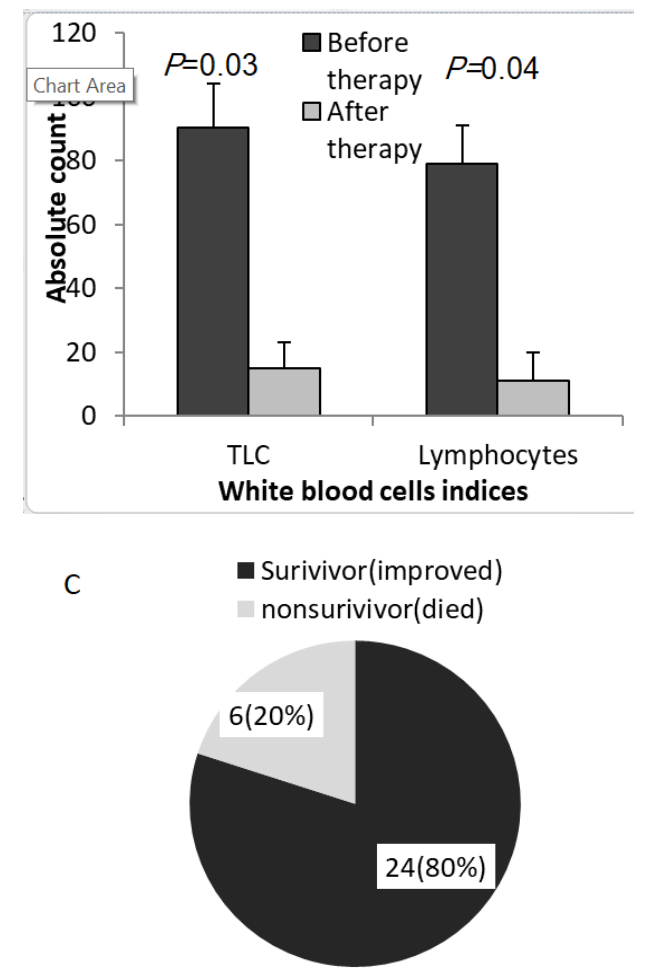

Figure 2. Patients chacracteristics. (a) Stratification of patients according to modified Rai staging, $9(30 \%)$ patients were classified as high risk CLL, 6 (20\%) intermediate risk CLL and 15 (50\%) low risk. (b) Level of leucocytic count before and after therapy ( $90.25 \pm 14.78$ vs. $14.85 \pm 8.16 ; P=0.03)$ and lymphocytic count (78.88 \pm 12.45 vs. $11.88 \pm 20.68$; $P=0.04$ ) (c) Outcome of patients with CLL Six patients in the study.

\section{CXCR4 level in CLL patients}

The level of CXCR4 significantly higher in patients' group in comparison to control group $(81.67 \pm 17.95 \%$ vs. $11.78 \pm 2.78 \% ; P<0.001)$ so these number is mean percentage of the flowcytometric measurement of CXCR4 from the gated bone marrow mononuclear cells Figure 3a, was insignificantly higher in females' patients in comparison to males' patients (82.37 \pm 8.75 vs. $81.36 \pm 10.87 ; P=0.89$ ).

Table 6 showed the expression of CXCR4 in the studied patients according to the modified Rai staging system. The level of CXCR4 was significantly higher in patients with high risk CLL (93.63 \pm 6.78$) P$ value $<0.001$ in comparison to patients with intermediate risk $(82.50 \pm 7.13)$ and patients with low risk (75.84 \pm 12.23$)$ Figure $3 \mathrm{~b}$. The therapeutic data and the response of the studied patients to FCR chemotherapy is presented in Table 5 . The FCR regimen was standard of care and first line ( $n=7 ; 23.3 \%)$. CVP (Cyclophosphamide, Vincristine, Prednisolone) regimen was added as second option $(n=2$; $6.7 \%)$. Majority of patients ( $n=21 ; 70 \%$ ) received combination of FCR as a first line and CVP regimens as a second line to achieve second remission. Median number of cycles was three cycles with range between one cycle and five cycles. As regarding response in such patients, it was noticed that 13 (43.3\%), 6 (20\%), and 11 (36.7\%) patients had complete response, partial response and progressive course, six patients in the study were deteriorated and died Figure $2 \mathrm{c}$.

The level of CXCR4 expression based on response of the studied patients to FCR chemotherapy is shown in table 6. It was noticed that level of CXCR4 was significantly higher in patients with progressive course $(91.63 \pm 6.98) P$ value $<0.001$ in comparison to patients with partial response $(83.11 \pm 5.55)$ and patients with complete response (70.11 \pm 4.44). Level of CXCR4 in patients based on outcome shown in Table 6.

It was noticed that CXCR4 had moderate positive correlation with Total leucocytic count $(r=0.45, \quad P=0.01)$ and relative count of lymphocyte in complete blood picture_( $r=0.40$, $P=0.01$ ), while insignificant correlations with other laboratory data were found Table 7. 
a

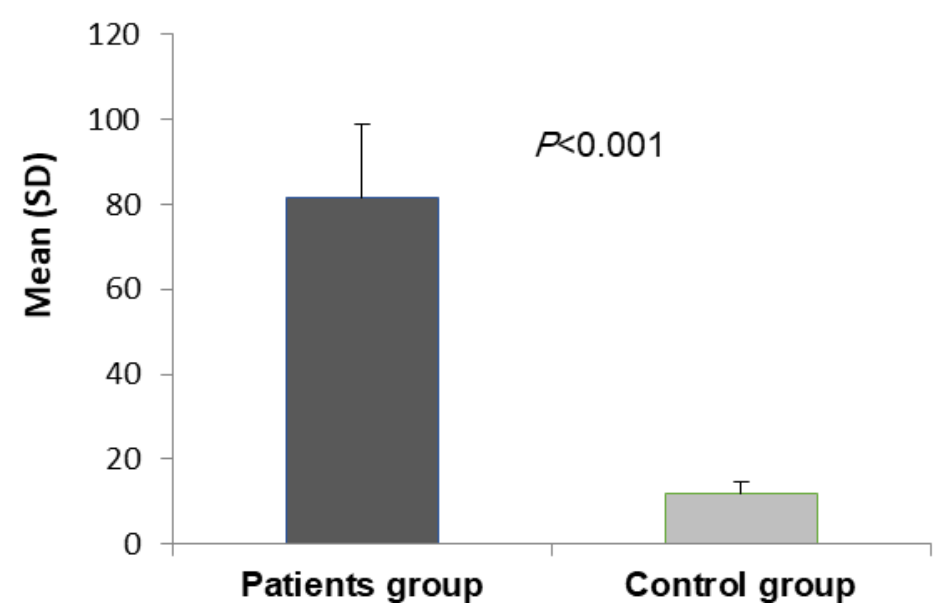

b

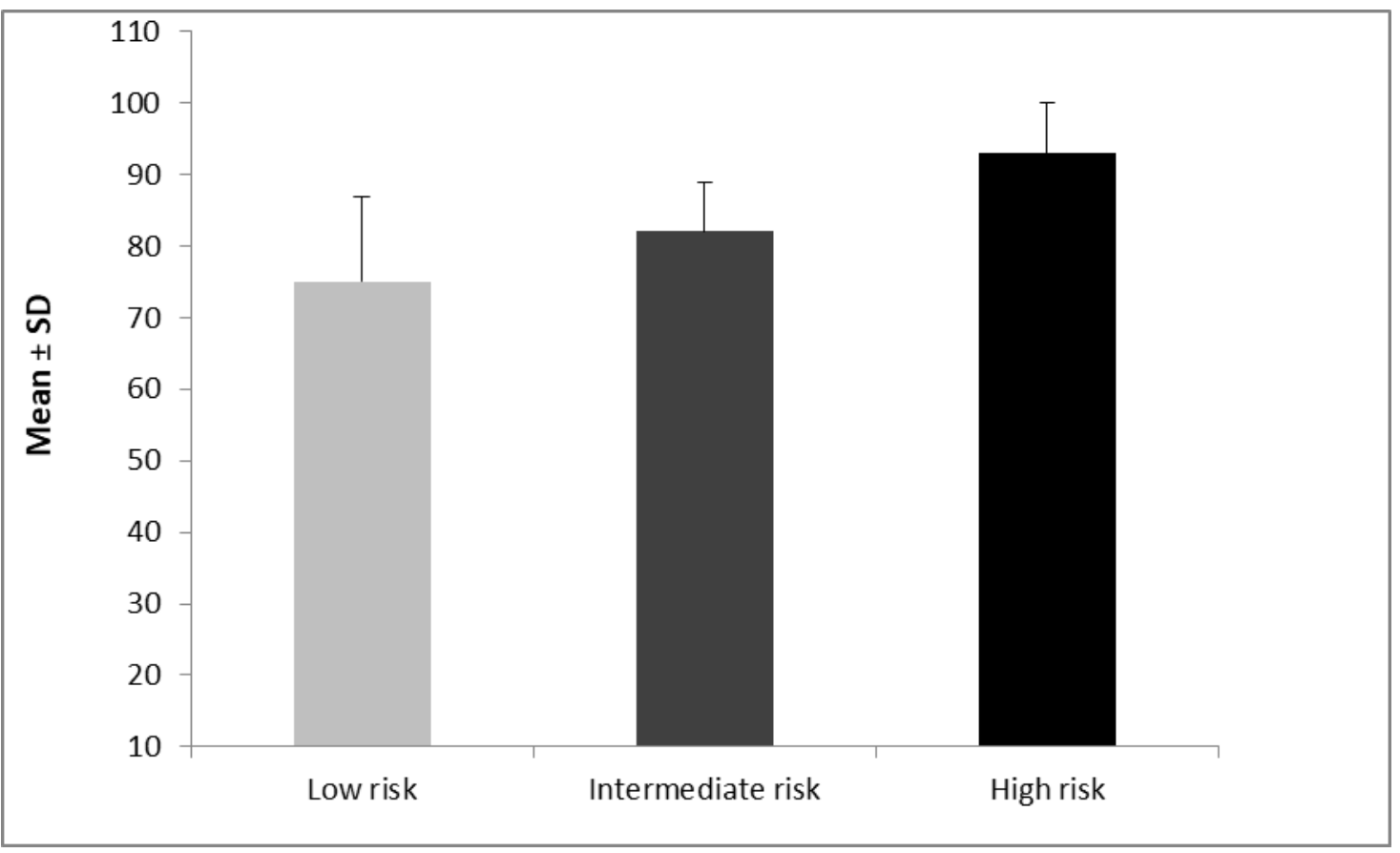

Figure 3. CXCR4 expression measurement. (a) Level of CXCR4 expression was significantly higher in patients in comparison to control group (81.67 \pm 17.95 vs. $11.78 \pm 2.78 ; P<0.001)$. (b) Level of CXCR4 according to modified Rai classification. There were significantly higher levels of CXCR4 in high risk CLL patients in comparison to intermediate and low risk $93.63 \pm 6.78$ vs. $82.50 \pm 7.13$ vs. $75.84 \pm 12.23 ; P<0.001 . P$ value is considered significant if $<0.05$. 
Table 5. Therapeutic data and outcome in studied groups.

\begin{tabular}{lc}
\hline & $\mathrm{N}=30$ \\
\hline Regimens & $7(23.3 \%)$ \\
FCR & $2(6.7 \%)$ \\
CVP & $21(70 \%)$ \\
Combined & $3(1-5)$ \\
Number of cycles & \\
Response to treatment & $13(43.3 \%)$ \\
Complete response & $6(20 \%)$ \\
Partial response & $11(36.7 \%)$ \\
Progressive course & \\
Outcome & $24(80 \%)$ \\
Survivor (improved) & $6(20 \%)$ \\
$\quad$ Non survivor (died) &
\end{tabular}

Table 6. CXCR4 expression in CLL patients with CLL.

\begin{tabular}{lcc}
\hline CXCR4 in Patients based on modified Rai Staging: & CXCR4 & $P$ value \\
\hline Low risk $(n=9)$ & $75.84 \pm 12.23$ & \\
Intermediate risk $(n=6)$ & $82.50 \pm 7.13$ & $<0.001$ \\
High risk $(n=15)$ & $93.63 \pm 6.78$ & \\
\hline CXCR4 in Patients based on treatment Response: & CXCR4 & $P$ value \\
\hline Complete response $(n=13)$ & $70.11 \pm 4.44$ & \\
Partial response $(n=6)$ & $83.11 \pm 5.55$ & $<0.001$ \\
Progressive course $(n=11)$ & $91.63 \pm 6.98$ & \\
\hline CXCR4 in Patients based on Outcome: & CXCR4 & $P$ value \\
\hline Improved $(n=13)$ & $80.89 \pm 5.09$ & $<0.001$ \\
Progressive $(n=11)$ & $91.63 \pm 6.98$ & \\
\hline
\end{tabular}

$P$ indicates significance of correlation and considered significant if $<0.05$.

CXCR4 level is percentage of the flowcytometric measurement of cxcr4 from the gated bone marrow mononuclear cells.

Table 7. Correlation of CXCR4 expression with other parameters in patients with CLL.

\begin{tabular}{llc}
\hline Parameters & rvalue & $P$ value \\
\hline Complete blood picture & $\mathrm{Nil}$ & -- \\
Hemoglobin $(\mathrm{g} / \mathrm{dl})$ & $\mathrm{Nil}$ & -- \\
Platelets $\left(\times 10^{3} / \mu \mathrm{l}\right)$ & 0.45 & 0.01 \\
White blood cells $\left(\times 10^{3} / \mu\right)$ & 0.33 & $\mathrm{NS}$ \\
Neutrophil $(\%)$ & 0.40 & 0.01 \\
Lymphocytes $(\%)$ & 0.11 & $\mathrm{NS}$ \\
Monocytes $(\%)$ & $\mathrm{Nil}$ & --- \\
Eosinophil $(\%)$ & 0.10 & $\mathrm{NS}$ \\
Reticulocyte $(\%)$ & &
\end{tabular}

.$r$, coefficient of correlation. $P$ value is considered non significant (NS) if $>0.05$ 


\section{Discussion}

CLL is the most common type of leukemia account for $40 \%$ of all leukemias and mainly affecting elderly, biological prognostic markers had provided new insights into the risk stratification of patients with CLL. ${ }^{16}$

CXCR4 is a chemokine $\mathrm{G}$ protein coupled receptor, present in different cell types, including normal B cells. CXCR4 is overexpressed in a variety of solid and hematological malignancies. ${ }^{4}$

In this case control study, we have studied through the expression of CXCR4 in 30 newly diagnosed CLL patients and 30 healthy control and evaluated the role of CXCR4 in pathophysiology of CLL, as well as their role in diagnosis of CLL and their effect on prognosis of CLL patients. Limitation of the present study were small sample size, short term follow-up of patients and other factors that might affect patient's outcome e.g. financial support, chemotherapy.

In this study, CLL was more predominant in males (76.7\%) and more common above 60 years old age group. It is consistently found that CLL is the disease of older age with median age 59years and male predominance. ${ }^{17-20}$

We found that constitutional symptoms (fatigue $80 \%$ and fever $46.7 \%$ ) are the most frequent symptom in patients followed by lymphadenopathy (43.3\%) and splenomegaly (40\%). Similarly as it was described in Catovsky et al ${ }^{21}$, who reported that the patients may present with localized or generalized lymphadenopathy, hepatosplenomegaly or constitutional symptoms. And also consistent with Rai and Jain, ${ }^{10}$ who reported that the patients commonly present with asymptomatic peripheral blood lymphocytosis or leukocytosis (predominantly lymphocytosis), lymphadenopathy, hepatosplenomegaly, bone marrow failure, and recurrent infections and often with autoimmune hemolytic anemia or autoimmune thrombocytopenia. On the contrary, Parker and Strout reported that up to $50 \%$ of CLL patients were asymptomatic mostly due to the frequent hematological follow up of patients in this study. $^{22}$
The present study showed that, the peripheral blood picture of the studied patients showed leukocytosis with absolute lymphocytosis. In agreement with our results Hallek et al, ${ }^{23}$ reported that in CLL there is leukocytosis with absolute lymphocytosis due to accumulation of malignant, mature clonal B lymphocytes in the blood, bone marrow and other lymphoid tissues. In this study, there were significant reduction in Leukocyte and Iymphocyte count after therapy. Same result was obtained by Atef et al, $^{24}$ who showed significantly decrease in leukocytosis and absolute lymphocytic count after therapy in infiltrative type.

This study revealed, some cases show mild to moderate anemia $20 \%$ and thrombocytopenia 33.3\%. In agreement with our results of Visco et al, and Rodrigues, et al. $^{25,26}$ who reported that anemia and thrombocytopenia may be observed in 15-30\% of patients. They generally result from bone marrow infiltration and become severe with progressive replacement of bone marrow by leukemic cells never the less the immune thrombocytopenia and hemolytic anemia that commonly associated with CLL.

Results demonstrated that, liver and kidney profiles were normal in the majority of studied cases, while LDH was significantly elevated. This finding was matched with recent study of Catovsky et al, ${ }^{27}$ who reported that CLL patients with high LDH has worse prognosis than those with normal LDH. LDH, a marker of cell turnover, also correlates with disease activity and worse prognosis.

The bone marrow aspirate of the studied patients showed that the most dominant cells were lymphocytes. Same results are reported by Bedu-Addo et al, and Richards, who showed that in CLL, there is replacement of normal marrow elements by leukemic B lymphocytes. It was noticed that out of the studied patients most of them are low risk. ${ }^{28,29}$ Because most of the patient are asymptomatic and these patients with early-stage disease Rai 0 , they should be monitored without therapy unless they have evidence of rapid disease progression.

Interestingly this present study revealed that CXCR4 was significantly higher in patients' 
group CD19+/CD5+/CD23+ positive in comparison to control group. These results were in concordance with the results of Li et al ${ }^{7}$,who reported that, in CLL, CXCR4 over expressed and CXCR4-expressing cancerous Bcells are attracted toward bone marrow stromal cells, which secrete high levels of CXCL12, resembling the homing of normal hematopoietic stem cells to bone marrow. CXCR4 is overexpressed in a variety of cancers including CLL, acute myeloid leukemia, myeloma, lymphomas, and solid tumors. ${ }^{4,30}$ The chemokine receptor CXCR4 is abnormally expressed in B-CLL cells, which exhibit the CD19+/CD5+/CD23+ phenotype.

The present study showed the percentage of CXCR4on the gated cells by flowcytometry were insignificantly higher in females' patients in comparison to male patients. Similarly, as what was reported by Sauvé et al that there were increase in CXCR4 in females. He explained that by a positive regulatory loop between the CXCR4/SDF-1 chemokine axis and estrogen receptor (ER)signaling pathways, which influence both ER- and CXCR4-dependent gene expression and ultimately tumor cell growth in vitro. $^{31}$

Same results are obtained by Barretina et $\mathrm{al}^{32}$ who reported that overexpression of CXCR4 in B-CLL cells has been associated with increased functional response to $\mathrm{CXCL} 12$ as measured by increased intracellular signaling, SDF-1-dependent migration.

Our finding is in agreement with Majid et al, $^{33}$ who showed that CXCR4 is increased in CLL patients and CXCR4 plays a role in the migration of B-cells from PB to $L N$ and BM. And this is critical in increasing their survival. Consequently, improved survival of B-cells increase can be linked with an aggressive and accelerated clinical course of CLL.

This study revealed that CXCR4 was significantly higher in patients with high risk CLL (Rai stage III-IV) in comparison to those with intermediate risk (Rai stage I-II) and those with low risk (Rai stage 0). Ghobrial et al showed that the expression of CXCR4 and CCR7 on B-CLL cells correlates with Rai stage. Also, these chemokine receptors may be down-regulated once malignant $B$ cells enter the lymph nodes ${ }^{34}$. On the contrary, Dürig et al reported that there is no difference between CXCR4 expression and disease stage or difference in mean CXCR4 expression when patients were grouped according to Rai's or Binet's stage of disease. ${ }^{30}$ Similarly, CXCR4 expression could not have prognostic impact at diagnosis. Although in the heterogeneous Binet A patient group, low CXCR4 expression was significantly associated with elevated LDH level at diagnosis, which may indicate high turnover rate of CLL cells. ${ }^{35}$ Also, CLL cells express an abundance of CXCR4 protein and doesn't correlate with disease stage which not matched with the patient results. ${ }^{32}$

It was noticed that level of CXCR4 was significantly lower in improved patients in comparison to those with progressive course. Same results was found in Peng,et al ${ }^{36}$, who reported that the CXCR4 overexpression was correlated with poor prognosis in CLL patients. The activation of CXCR4 induces cell trafficking and homing of malignant cells to the BM and lymph nodes, where CXCL12 is highly expressed, leading to retention of these cells in a microenvironment that provides growth signals, induces proliferation, and contributes to drug resistance, leading to poor prognosis and relapse. $^{37,38}$

In this study, CXCR4 had moderate positive correlation with TLC and lymphocytes. These results are in agreement with Burger,et al, ${ }^{39}$ who showed that there was clear correlation found between CXCR4 expression and both circulating leukocytes and CD19+/CD5+ cell count, and this lead us to hypothesis that CXCR4 could play a role in protecting malignant B-CLL cells from apoptosis.

\section{Author Contributions}

WE, DN and ES proposed and designed the study. ES and MS carried the laboratory and experimental work. MA and MS followed patients and collected clinical data. WE, MA and MS wrote the paper. ES and $\mathrm{DN}$ revised the paper.

\section{Declaration of Conflicting Interests}

The author(s) declared no potential conflicts of interest with respect to the research, authorship, and/or publication of this article. 


\section{Funding}

This research did not receive any specific grant from funding agencies in the public, commercial, or notfor-profit sectors.

\section{Ethical approval}

The study protocol was reviewed and approved by the Committee of Medical Ethics, Faculty of Medicine, Assiut University.

\section{Informed consent}

A signed consent form was obtained from each study participant.

\section{References}

1. Chiorazzi N, Rai KR, Ferrarini M. (2005) Chronic lymphocytic leukemia. New England Journal of Medicine. 352(8):804-815.

2. Slager SL, Kay NE. (2009) Familial chronic lymphocytic leukemia: what does it mean to me? Clinical Lymphoma and Myeloma. 9:S194-S197.

3. Bianchi S, Dighiero G, Pritsch O. (2012) Selected topics in chronic lymphocytic leukemia pathogenesis. Chronic Lymphocytic Leukemia: InTech; 2012.

4. Kashyap MK, Amaya-Chanaga Cl, Kumar D, et al. (2017) Targeting the CXCR4 pathway using a novel anti-CXCR4 IgG1 antibody (PF-06747143) in chronic lymphocytic leukemia. Journal of hematology \& oncology. 10(1):112.

5. Müller A, Homey B, Soto $H$, et al. (2001) Involvement of chemokine receptors in breast cancer metastasis. nature. 410(6824):50.

6. Burger M, Hartmann T, Krome M, et al. (2005) Small peptide inhibitors of the CXCR4 chemokine receptor (CD184) antagonize the activation, migration, and antiapoptotic responses of CXCL12 in chronic lymphocytic leukemia B cells. Blood. 106(5):1824-1830.

7. Zhou B, Lu Y, Hajifathalian K, et al. (2016) Worldwide trends in diabetes since 1980: a pooled analysis of 751 population-based studies with $4 \cdot 4$ million participants. The Lancet. 387(10027):15131530.

8. Hamblin TJ, Orchard JA, Ibbotson RE, et al. (2002) CD38 expression and immunoglobulin variable region mutations are independent prognostic variables in chronic lymphocytic leukemia, but CD38 expression may vary during the course of the disease. Blood. 99(3):1023-1029.

9. Eichhorst B, Robak T, Montserrat E, et al. (2015) lymphocytic leukaemia: ESMO Clinical Practice
Guidelines for diagnosis, treatment and follow-up. Annals of Oncology. 26(suppl_5):v78-v84.

10. Rai KR, Jain P. (2016) Chronic lymphocytic leukemia (CLL)-T hen and now. American journal of hematology. 91(3):330-340.

11. Hallek M, Cheson BD, Catovsky D, et al. (2018) iwCLL guidelines for diagnosis, indications for treatment, response assessment, and supportive management of CLL. Blood. 131(25):2745-2760.

12. Hus I, Roliński J. (2015) Current concepts in diagnosis and treatment of chronic lymphocytic leukemia. Contemporary Oncology. 19(5):361.

13. Bain BJ, Lewis SM. (2006) Preparation and staining methods for blood and bone marrow films. Dacie and Lewis Practical Haematology. 2006:59.

14. Landgren O, Albitar M, Ma W, et al. (2009) B-cell clones as early markers for chronic lymphocytic leukemia. New England Journal of Medicine. 360(7):659-667.

15. Bain BJ, Bates I, Laffan MA, et al (20) Practical Haematology: Expert Consult: Online and Print. Elsevier Health Sciences; 2016.

16. Yegin ZA, Özkurt ZN, Yağcı M. Free light chain: a novel predictor of adverse outcome in chronic lymphocytic leukemia. European journal of haematology. 2010;84(5):406-411.

17. Mukiibi J, Paul B, Nyirenda C, et al. (2004) Chronic lymphocytic leukaemia (CLL) in Central Africans. 2004.

18. Gogia A, Sharma A, Raina V, et al. (2012) Assessment of 285 cases of chronic lymphocytic leukemia seen at single large tertiary center in Northern India. Leukemia \& Iymphoma. 53(10):19611965.

19. Barr PM, Saylors GB, Spurgeon SEF, et al. (2014) Phase 2 trial of GS-9973, a selective syk inhibitor, and idelalisib (idela) in chronic lymphocytic leukemia (CLL) and non-Hodgkin lymphoma (NHL). American Society of Clinical Oncology; 2014.

20. Maurer C, Hallek M. (2013) Chronic lymphocytic leukemia. Deutsche medizinische Wochenschrift (1946). 138(42):2153-2166.

21. Catovsky D, Richards S, Matutes E, et al. (2007) Assessment of fludarabine plus cyclophosphamide for patients with chronic lymphocytic leukaemia (the LRF CLL4 Trial): a randomised controlled trial. The Lancet. 370(9583):230-239.

22. Parker TL, Strout MP. (2011) Chronic lymphocytic leukemia: prognostic factors and impact on treatment. Discovery medicine. 11(57):115-123.

23. Hallek M, Cheson BD, Catovsky D, et al. (2008) Guidelines for the diagnosis and treatment of chronic lymphocytic leukemia: a report from the 
International Workshop on Chronic Lymphocytic Leukemia updating the National Cancer InstituteWorking Group 1996 guidelines. Blood. 111(12):5446-5456.

24. Atef B, Azmy E, Aladle D, et al. (2019) The prevalence and prognostic significance of autoimmune cytopenias in a cohort of Egyptian patients with chronic lymphocytic leukemia. Hematology/oncology and stem cell therapy. 12(2):97-104.

25. Visco C, Cortelezzi A, Moretta F, et al. (2014) Autoimmune cytopenias in chronic lymphocytic leukemia at disease presentation in the modern treatment era: is stage C always stage C? Leukemia \& lymphoma. 55(6):1261-1265.

26. Rodrigues CA, Gonçalves MV, Ikoma MRV, et al. (2016) Diagnosis and treatment of chronic lymphocytic leukemia: recommendations from the Brazilian Group of Chronic Lymphocytic Leukemia. Revista brasileira de hematologia e hemoterapia. 38(4):346-357.

27. Catovsky D, Else M, Oscier D. (2019) The Clinical Presentation of CLL. Chronic Lymphocytic Leukemia: Springer; 39-50.

28. Bedu-Addo G, Amoako YA, Bates I. (2013) The Role od Bone Marrow Aspirate and Trephine Samples in Haematological Diagnoses in Patients Referred to a Teaching Hospital in Ghana. Ghana medical journal. 2013;47(2):74-78.

29. Richards S. (1999) Chemotherapeutic options in chronic lymphocytic leukemia: a meta-analysis of the randomized trials. CLL Trialists' Collaborative Group. Journal of the National Cancer Institute. 91(10).

30. Dürig J, Schmücker U, Dührsen U. (2001) expression of chemokine receptors in B cell malignancies. Leukemia. 15(5):752.
31. Sauvé K, Lepage J, Sanchez M, et al. (2009) Positive feedback activation of estrogen receptors by the CXCL12-CXCR4 pathway. Cancer research. 69(14):5793-5800.

32. Barretina J, Junca J, Llano A, et al. (2003) CXCR4 and SDF-1 expression in B-cell chronic lymphocytic leukemia and stage of the disease. Annals of hematology. 82(8):500-505.

33. Majid A, Lin TT, Best G, et al. (2011) CD49d is an independent prognostic marker that is associated with CXCR4 expression in CLL. Leukemia research. 35(6):750-756.

34. Ghobrial IM, Bone ND, Stenson MJ, et al. (2004) Expression of the chemokine receptors CXCR4 and CCR7 and disease progression in B-cell chronic lymphocytic leukemia/small lymphocytic lymphoma. Paper presented at: Mayo Clinic Proceedings2004.

35. Rossi D, Zucchetto A, Rossi FM, et al. (2008) CD49d expression is an independent risk factor of progressive disease in early stage chronic lymphocytic leukemia. haematologica. 93(10):15751579.

36. Peng S-B, Zhang X, Paul D, et al. (2016) Inhibition of CXCR4 by LY2624587, a fully humanized antiCXCR4 antibody induces apoptosis of hematologic malignancies. PloS one. 2016;11(3):e0150585.

37. Bollongino $R$, Nehlich $O$, Richards $M P$, et al. (2013) 2000 years of parallel societies in Stone Age Central Europe. Science. 342(6157):479-481.

38. Burger JA. (2011) Nurture versus nature: the microenvironment in chronic lymphocytic leukemia. ASH Education Program Book. 2011;2011(1):96-103.

39. Burger JA, Tsukada N, Burger M, et al. (2000) Blood-derived nurse-like cells protect chronic lymphocytic leukemia B cells from spontaneous apoptosis through stromal cell-derived factor-1. Blood. 96(8):2655-2663. 